\title{
Eight-Week Outcomes of Ledipasvir/Sofosbuvir in Noncirrhotic Treatment-Naive Patients with Hepatitis C: Analysis of Pharmacy-Based Data
}

\author{
Jennifer Andres, PharmD, BCPS; Stephen Lott, PharmD, MS, CSP; and Kamran Qureshi, MD
}

\begin{abstract}
BACKGROUND: Clinical trials have demonstrated that 8 weeks of ledipasvir and sofosbuvir (LDV/SOF) achieved high rates of sustained virologic response at 12 weeks (SVR12) in patients with hepatitis C viral (HCV) genotype 1 infection. The effectiveness of this combination was noted to be robust in treatment-naive noncirrhotic patients and in patients with an HCV viral load of $<6$ million IU $/ \mathrm{mL}$ before treatment. Generalizability of these results to community clinical practice, however, was advised with caution due to the variability of staging methods, fluctuating nature of viral loads, lack of prospective trials, and real-life confirmation.
\end{abstract}

OBJECTIVE: To evaluate the efficacy, defined as SVR12, of LDV/SOF in a real-world setting.

METHODS: Patients met inclusion criteria if given 8 weeks of LDV/SOF by a specialty pharmacy from 0ctober 2014 to 0ctober 2015 and if SVR12 was assessed after therapy completion. Clinical outcomes data were obtained from the pharmacy database.

RESULTS: Of the 6,391 prescriptions of LDV/SOF received by the pharmacy, $3,648(57 \%)$ were covered by insurance, and among them, only $511(14 \%)$ were for an 8-week regimen. SVR12 data were available for $380(74 \%)$ patients who completed an 8-week regimen. 230 different prescribers wrote prescriptions, and 57 different insurance plans approved the 8-week regimen. The majority $(74 \%)$ of patients were followed by gastroenterology clinics. The 380 patients included in the analysis were all treatment-naive HCV genotype 1 patients. Overall, SVR12 was achieved in $97 \%$ of patients, while 10 patients relapsed. The SVR12 rates were lower (93\%) in patients with stage 3 fibrosis, particularly in African Americans (29 of 35: 83\%).

CONCLUSIONS: Outcomes were favorable for the 8-week use of LDV/SOF in a noncontrolled real-world setting in treatment-naive noncirrhotic patients with a baseline viral load $<6$ million $\mathrm{IU} / \mathrm{mL}$. Use of this approach in African Americans with evidence of advanced fibrosis should be avoided.

J Manag Care Spec Pharm. 2018;24(1):23-28

Copyright $\odot 2018$, Academy of Managed Care Pharmacy. All rights reserved.

\section{What is already known about this subject}

Initial evidence for an 8-week treatment duration of ledipasvir/ sofosbuvir comes from a post hoc analysis of a clinical trial. - Real-world analyses have agreed with the hepatitis C viral (HCV) RNA threshold of $<6$ million IU/mL to qualify for 8 weeks of treatment.

Current guidelines recommend that patients with HCV RNA $<6$ million IU/mL can use ledipasvir/sofosbuvir for 8 weeks if they are not African American or HIV coinfected.

\section{What this study adds}

This study provided results that were consistent with current guideline recommendations.

Sustained virologic response at 12 weeks was high in the African American patient population, except in patients with stage 3 fibrosis, and also high in the HIV-coinfected patient population.

C hronic hepatitis C virus (HCV) infection affects an estimated 3-4 million people in the United States and an estimated 80-185 million people worldwide. ${ }^{1,2} \mathrm{HCV}$ is the leading cause of end-stage liver disease, hepatocellular carcinoma (HCC), and liver transplant in the United States. ${ }^{3}$ Deaths resulting from HCV infection have continued to rise, with HCV complications killing more Americans than 60 other infections combined. ${ }^{4}$ Treatment has advanced over the last decade with the approval of direct-acting antivirals (DAA), which improve treatment outcomes and reduce drugrelated toxicity compared with previously used regimens. ${ }^{5}$ Studies have shown that eradicating HCV decreases the risk of progression to end-stage liver disease and improves mortality.

The most common HCV genotype in the United States is genotype 1 (GT1). ${ }^{1}$ While other countries may have differing predominant genotypes, GTl infection exists worldwide. The rate of sustained virologic response at 12 weeks (SVR12) after treatment completion- the clinical marker for HCV treatment success or cure-is over $90 \%$ with DAA regimens in treatmentnaive patients with GTl infection. The American Association for the Study of Liver Disease (AASLD) and Infectious Disease Society of America (IDSA) have collaborated to create clinical guidelines that categorize recommendations by genotype, treatment experience, and presence or absence of cirrhosis. ${ }^{7}$ Current guidelines from the European Association for the Study of the Liver (EASL) also address treatment in a similar way. ${ }^{8}$

The combination of ledipasvir and sofosbuvir (LDV/SOF) was first approved for use in the treatment of chronic GT1 HCV by the U.S. Food and Drug Administration (FDA) in $2014 .{ }^{9}$ It was the first commercially available once-daily combination tablet for GTl infections. ${ }^{10}$ Initial evidence for an 8 -week treatment duration arose from the open-label, phase 2 LONESTAR-1 trial, which included noncirrhotic patients 
with GT1 infection who were treatment naive or previously treated with a protease-inhibitor regimen. A small subgroup of treatment-naive patients $(n=20)$ received 8 weeks of LDV/SOF, and $95 \%$ of patients obtained SVR $12 .{ }^{11}$

A larger phase 3 trial (ION-3) evaluated noncirrhotic treatment-naive patients with GTl on LDV/SOF with or without ribavirin (RBV) for 8 or 12 weeks. ${ }^{12}$ The patient population was mostly Caucasian males with a mean age in the mid-50s (range 20-75). There were no statistically significant differences in SVR12 between groups (8-week LDV/SOF 94\%; 8-week LDV/SOF/RBV 93\%; 12-week LDV/SOF 95\%), and noninferiority was met for the 8-week LDV/SOF regimen. While noninferiority was established, a larger number of relapses was identified in the 8-week treatment groups (11 patients [5\%] 8-week LDV/SOF; 9 patients [4\%] 8-week LDV/SOF/RBV; and 3 patients [1\%] 12-week LDV/SOF). Because of the small number of relapses in each arm, no baseline characteristics or response variables could be associated with an increased risk of relapse..$^{12}$ A post hoc analysis determined that patients with a baseline viral load of $<6$ million $\mathrm{IU} / \mathrm{mL}$ could be treated with 8 weeks of therapy without concern for relapse.

Based on the results of these trials, the FDA recommended 8 weeks of LDV/SOF, and the AASLD/IDSA initially included this regimen in their clinical guidelines as a consideration for treatment-naive GTl infection with a low HCV viral load, defined as the post hoc viral load of $<6$ million IU/mL in ION-3. Criticism followed the FDA approval and AASLD/IDSA recommendation because the viral load threshold was determined from the uncontrolled post hoc subgroup analysis, which was underpowered and could reflect a statistical artifact. ${ }^{13}$ Citing conflicting evidence, the 8-week recommendation was removed, but guidelines noted that it could be considered in specific situations with expert consultation. ${ }^{7}$

As HCV treatment experience grew, guidelines were updated and suggested that limiting treatment duration to 8 weeks can be considered in noncirrhotic patients with a baseline viral load of $<6$ million $\mathrm{IU} / \mathrm{mL}$, provided that they are not coinfected with human immunodeficiency virus (HIV) or of African-American descent. ${ }^{7,14,15}$ Current EASL guidelines advise that the duration of LDV/SOF could be shortened to 8 weeks in treatment-naive noncirrhotic patients with GTl, although caution is advised in those patients with F3 fibrosis, pending confirmation of these results in real-world studies. ${ }^{8}$

Unfortunately, cost plays a role in the decision to treat HCV infection and can be a barrier to accessing treatment. ${ }^{16,17}$ Despite the high cost of DAAs, the treatment of patients with GTl infections who have not been previously treated is considered to be a cost-effective strategy. ${ }^{18,19}$ Limiting treatment duration to 8 weeks with LDV/SOF in certain patients can be used as a way to lower costs without sacrificing efficacy. Several real-world analyses have been performed to add support for an 8 -week treatment regimen and have reported high SVR rates for patients treated for 8 weeks with $\mathrm{LDV} / \mathrm{SOF} \pm \mathrm{RBV} .{ }^{20-27}$ One of these real-world studies, Kowdley et al. (2017), ${ }^{27}$ combined data from a specialty pharmacy in the northeastern United States, a managed care organization in southern California, and a physician group practice in Germany.

The purpose of our study was to evaluate the subset of data originating from the specialty pharmacy in the Kowdley et al. study to assess the efficacy of SOF/LDV in a specialty pharmacy setting with a wide range of prescribers, practice settings, and patients.

\section{Methods}

The study population included patients with GTl HCV infection who had a prescription for LDV/SOF processed by a specific specialty pharmacy. This specialty pharmacy works with multiple prescribers and institutions and serves a large population residing in the northeastern region of the United States, which includes Pennsylvania, New Jersey, Delaware, and Maryland. The pharmacy assists prescribers with prior authorizations, patient education, and timely delivery of prescriptions to patients. All services are provided with consistent communication with prescribers.

The specialty pharmacy used an electronic database to track patient outcomes. Data were collected retrospectively for all treatment-naive patients with GTl who were provided 8 weeks of LDV/SOF by the pharmacy from October 2014 to October 2015 and had completed therapy for at least 12 weeks. Evaluation of outcomes occurred only for patients with available SVR12 results. The primary outcome was the percentage of patients achieving SVR12, defined by a negative HCV RNA $(<15 \mathrm{IU} / \mathrm{mL})$ by polymerase chain reaction assessed at 12 weeks following the completion of the 8-week regimen of LDV/SOF. Treatment failure was defined as an inability to obtain SVR12, either because of relapse or positive viral load during treatment. Relapse was defined as positive HCV RNA (>15 IU/ $\mathrm{mL}$ ) after the completion of the 8-week regimen of LDV/SOF.

The clinical characteristics recorded for each patient included age, gender, ethnicity, body weight, HIV coinfection status, presence or absence of renal disease, diabetes or HCC, and physician practice type. Dates of pretreatment viral load testing, liver fibrosis assessment, and therapy initiation were collected to assess the amount of time between evaluation and start of therapy. Collected clinical values included genotype, pretreatment viral load, and documented absence of cirrhosis, designated as noncirrhotic by provider or with a Metavir fibrosis stage 0-3 (FO-F3, where F0 indicates a normal liver and F4 indicates a cirrhotic liver), if known. Fibrosis stage and absence of cirrhosis were determined at the discretion of the treating physician and could include liver biopsy, serum biomarkers, or vibration-controlled elastography.

Evaluation of clinical characteristics, including determination of cirrhosis status, was at the discretion of the prescribing physician 


\begin{tabular}{|c|c|c|}
\hline & \multicolumn{2}{|c|}{$\mathrm{N}=380$} \\
\hline Age, median (range), years & \multicolumn{2}{|c|}{$58 \quad(22-82)$} \\
\hline \multicolumn{3}{|l|}{ Gender, $\mathrm{n}$} \\
\hline Male & \multicolumn{2}{|c|}{210} \\
\hline Female & \multicolumn{2}{|c|}{170} \\
\hline \multicolumn{3}{|l|}{ Race, n (\%) } \\
\hline Caucasian & 225 & (59) \\
\hline African American & 116 & $(31)$ \\
\hline Others & 39 & $(10)$ \\
\hline \multicolumn{3}{|l|}{ Genotype, n (\%) } \\
\hline GT la & 264 & (69) \\
\hline GT lb & 89 & (24) \\
\hline GT 1 or $l a / l b$ & 27 & $(7)$ \\
\hline \multicolumn{3}{|l|}{ Metavir stage, $\mathrm{n}(\%)$} \\
\hline F0 (stage 0) & 57 & $(15)$ \\
\hline Fl (stage 1) & 64 & (17) \\
\hline F2 (stage 2) & 103 & $(27)$ \\
\hline F3 (stage 3) & 86 & $(23)$ \\
\hline Noncirrhotics, $\mathrm{n} \%$ & 70 & (18) \\
\hline $\begin{array}{l}\text { Baseline HCV RNA, mean (range), } \\
\text { IU/mL }\end{array}$ & \multicolumn{2}{|c|}{$\begin{array}{l}1,630,000 \text { million } \\
(493-5,960,000)\end{array}$} \\
\hline $\begin{array}{l}\text { Gap between viral load testing and } \\
\text { initiation of therapy, median (range), days }\end{array}$ & \multicolumn{2}{|c|}{$100 \quad(1-176)$} \\
\hline$H C V=$ hepatitis $C$ virus. & & \\
\hline
\end{tabular}

and insurance requirements. Treatment completion and compliance was confirmed by prescription-dispensing records. This study was conducted in compliance with institutional review board/human subjects research committee requirements.

\section{Results}

\section{Prescription Data}

During the first 12 months after approval of LDV/SOF (October 2014-October 2015), 6,391 prescriptions containing LDV/SOF were received by the specialty pharmacy, and 3,648 (57\%) were approved by insurance companies for payment. Among the covered LDV/SOF prescriptions, the majority (86\%) were for regimens other than the 8-week duration. Five hundred and eleven prescriptions for 8 weeks of LDV/SOF were approved for payment and dispensed to patients. All patients completed 8 weeks of treatment, evidenced by refill-dispensing history. Eight-week prescriptions were written by 230 different prescribers, with 57 different insurance companies approving the regimen for dispensing.

\section{Study Cohort}

Results are presented for patients who had SVR12 data logged into the database. There are an additional 73 patients in this analysis, including those classified as noncirrhotic, as compared with the patients in the parent study from the specialty pharmacy $(n=307) .{ }^{27}$ The additional 5 patients that were

\begin{tabular}{|c|c|c|c|c|c|c|}
\hline & $\begin{array}{c}\text { Total } \\
\text { Population }\end{array}$ & F0 & F1 & F2 & F3 & Noncirrhotic \\
\hline \multicolumn{7}{|l|}{ erall } \\
\hline tal & 380 & 57 & 64 & 103 & 86 & 70 \\
\hline ailures & 10 & 0 & 0 & 1 & 6 & 3 \\
\hline VR12 & 370 & 57 & 64 & 102 & 80 & 67 \\
\hline \multicolumn{7}{|c|}{ ucasians } \\
\hline tal & 225 & 43 & 44 & 57 & 36 & 45 \\
\hline ailures & 3 & 0 & 0 & 1 & 0 & 2 \\
\hline VRl2 & 222 & 43 & 44 & 56 & 36 & 43 \\
\hline \multicolumn{7}{|c|}{ ican Americans } \\
\hline otal & 116 & 11 & 19 & 34 & 37 & 15 \\
\hline ailures & 7 & 0 & 0 & 0 & 6 & 1 \\
\hline VR12 & 109 & 11 & 19 & 35 & 31 & 14 \\
\hline \multicolumn{7}{|l|}{ hers } \\
\hline tal & 39 & 3 & 2 & 11 & 13 & 10 \\
\hline ailures & 0 & 0 & 0 & 0 & 0 & 0 \\
\hline VR12 & 39 & 3 & 2 & 11 & 13 & 10 \\
\hline
\end{tabular}

SVR12 = sustained virologic response at 12 weeks.

excluded from the parent study were missing variables, such as exact baseline viral load. SVR12 data were available from 380 (74\%) patients who completed the 8-week regimen of LDV/ SOF. Patients who did not have SVR12 data available $(n=131)$ were not included in the outcomes assessment. Among the patients with SVR12 data available, 187 different prescribers provided care for the patients. Prescribers included physicians, physician assistants, and nurse practitioners. The majority (74\%) of 380 patients were treated in gastroenterology clinics, followed by hepatology clinics (19\%) and infectious diseases clinics (8\%). On average, each hepatologist wrote 7.1 prescriptions, while gastroenterologists wrote 1.7 prescriptions, and infectious disease physicians wrote 1.6 prescriptions for the 8-week regimen.

Baseline characteristics of the patient population are shown in Table 1. Most of the patients were male (55\%) and Caucasian (59.5\%). All patients were treatment naive and had GTl infection. All patients had a pretreatment viral load $<6$ million IU/mL, consistent with AASLD/IDSA guidelines. ${ }^{7}$ Staging of liver disease was performed by various methods based on the discretion of the prescribing practitioner, with the most common (40\%) method being a FibroSURE (Laboratory Corporation of America, Raritan, NJ) blood test. Liver biopsy was performed in $24 \%$ of patients. The median gap between fibrosis assessment and initiation of therapy was 9 weeks in FibroSURE and 49 weeks in liver biopsy. No patients had cirrhosis. Fibrosis stage was not available for $18 \%$ of patients, but they were included in the analysis, since they were classified as noncirrhotic by their prescribers. Only 16 of 380 (4.2\%) patients were HIV coinfected. 


\section{Virologic Outcomes}

Overall, 97\% of patients achieved SVR12 (Table 2), with only 10 patients who failed treatment. SVR12 was achieved by 99\% of Caucasians (222/225) and 94\% of African Americans (109/116). All patients identified as a race other than Caucasian or African American achieved SVR12. All patients with F0 $(\mathrm{n}=57)$ and F1 $(\mathrm{n}=64)$ achieved SVR12. There was only 1 treatment failure among patients with stage F2 $(n=103)$. Most treatment failures occurred in patients with F3 (SVR12 93\%, $\mathrm{n}=86)$. There were 6 failures in this subgroup, and all were African Americans. Thus, SVR12 was achieved by 83\% $(n=31)$ of African Americans with F3 disease. Among the 70 patients labeled noncirrhotic, 3 failed treatment. SVR12 was consistent with race in this group as well: Among Caucasians, SVR12 was 96\%, and among African Americans, SVR12 was 93\%. In the HIV-coinfected population, all but 1 patient achieved SVR12 (94\%; data not shown).

Of interest, $28 \%$ of the 8 -week LDV/SOF prescriptions were initially written for 12-week treatment duration. The treatment duration was changed to 8 weeks, either by the provider or because of insurance coverage. Among those who were initially prescribed 12 weeks of therapy and completed 8 weeks of therapy, 93\% achieved SVR12.

All 10 treatment failures were patients who relapsed after completion of 8 weeks of LDV/SOF therapy. Only 1 patient was HIV coinfected. This patient was initially prescribed a 12 -week regiman, which was mandated to 8 weeks in duration by insurance. Fibrosis stage was evaluated in 8 patients by serologic testing, in 1 patient by liver biopsy, and was not performed in 1 patient. The average baseline viral load was 2,301,553 (range: 16,1736-5,310,000). All but 4 patients who failed treatment had a fibrosis stage of F3 disease. No patients with treatment failure were reported to have a history of HCC. A change of treatment duration from 12 weeks occurred in 7 patients who failed 8 weeks of therapy. Of these, 3 prescribers discontinued therapy at 8 weeks, and 4 insurance prescribers mandated 8 weeks of therapy.

\section{Discussion}

In this analysis of specialty pharmacy data of treatment-naive GT1 infected noncirrhotic patients within a noncontrolled realworld setting, 97\% of the 380 patients who received 8 weeks of LDV/SOF achieved SVR12. This analysis included 187 different practitioners across the northeastern region of the United States, involving a wide range of patients and practice strategies across several specialties. All patients who received 8 weeks of LDV/SOF had a pretreatment viral load of $<6$ million IU/mL, consistent with FDA labeling, current AASLD/IDSA recommendations, and current EASL guidelines. ${ }^{7,8,10}$ Treatment duration of 8 weeks was selected at the discretion of the treating physician and insurance providers, increasing the applicability of this data since insurance providers issue authorizations for
LDV/SOF in the United States. There were no differences in the SVR12 rate in patients who received a shorter duration of therapy mandated by an insurance provider as compared with those who were initially ordered to receive therapy for 8 weeks.

These results are similar to those seen in clinical trials, including ION-3, which has demonstrated generalizability based on the results of other real-world cohort studies. ${ }^{22-27}$ These results are also consistent with the parent study from which some of these data originate. ${ }^{27}$ The overall SVR12 rate was $98.1 \%$ (622/634) for the entire population, similar to the SVR12 seen in this cohort. The SVR12 rate among African Americans was $>95 \%$ in the full study, similar to the $94 \%$ in this cohort. ${ }^{27}$ For patients with F3, the SVR12 rate was $94.1 \%$ in the entire population and $93 \%$ in this cohort. Notably, all patients with F3 who failed therapy were African American. Based on these results, special caution is advised for the African-American population, especially in those with more significant hepatic disease. There were no treatment failures in HIV-coinfected patients given 8 weeks of treatment with baseline HCV RNA viral level $<6$ million IU/mL in the entire population. ${ }^{27}$ Only 1 coinfected patient failed treatment in our population, with stage 3 fibrosis.

Treatment failure rate was low in this cohort, with only 10 patients experiencing treatment failure. Important predictors of a lower treatment response were race and presence of stage 3 fibrosis. A higher percentage of African-American patients failed treatment compared with Caucasians, despite a smaller number of African Americans in the overall population. The SVR12 rates were also lower (93\%) in patients with stage 3 fibrosis, particularly in African Americans (31/37: 83\%). This result is consistent with guideline recommendations to avoid an 8-week treatment duration in this patient population.

Despite the recommendation for HIV-coinfected patients to receive 12 weeks of LDV/SOF, our study showed positive outcomes after an 8-week treatment duration in the HIVcoinfected population. All but 1 coinfected patient attained SVR12. Notably, this patient had stage 3 fibrosis. Caution is advised in interpreting this finding, since only 16 patients were coinfected with HIV; however, a 100\% SVR12 rate was observed within the parent study population. ${ }^{27} \mathrm{~A}$ larger cohort of coinfected patients would help determine if HIV-coinfected patients can be effectively treated as mono-infected patients.

\section{Limitations}

This analysis is limited by the retrospective design and lack of a comparator group. Data were gathered from a database used by a specialty pharmacy, so we relied on provided clinical information. Although the pharmacists at the specialty pharmacy communicated closely with the prescribers for missing clinical information, the database was not complete, as evidenced by the 131 patients who did not have documented SVR12 results. Efforts were made to collect the maximum amount of the missing data by call backs to the providers. Also, comorbidities 
such as diabetes, renal disease, and coinfections may not be accurate in the database, since it may not have been captured in the prescription from the provider. Accurate representation of coinfection data would be important to record to determine if HIV coinfected patients can receive 8 weeks of LDV/SOF.

Despite challenges in obtaining information, this study assessed a larger number of patients from the specialty pharmacy than the population evaluated in the parent study $(\mathrm{n}=307) .{ }^{27}$ Seventy (18\%) patients were labeled noncirrhotic, since fibrosis staging information was not provided in the prescription. Three patients in this group failed treatment, so it would be helpful to know the baseline fibrosis stage and whether this had an effect on treatment failure.

Specialty pharmacies may not fill all prescriptions for each patient and so may have incomplete information regarding potential drug interactions, such as patients with prescriptions for acid-suppressing agents, which may negatively affect virologic response to LDV/SOF. Nevertheless, it is assumed that patient education on drug interactions was provided to all patients as is standard in this specialty pharmacy's practice.

While daily adherence and adverse events were not addressed in this analysis, all patients who initiated 8 weeks of treatment with LDV/SOF completed therapy based on the dispensing history of on-time refills. Some patients who failed therapy could have been nonadherent despite obtaining on-time refills. This is an accurate representation of real-world communication between a pharmacy who serves different health care systems and practitioners across a wide geographical area.

Despite these limitations, this study has a comparable SVR12 rate to that observed in clinical trials, even though it occurred in a real-world, noncenter, and noncontrolled community practice setting.

Currently, the LDV/SOF combination is 1 of 2 treatments that can be administered with success in specific patients for an 8 -week treatment duration. It is important to identify the clinical characteristics - treatment naive with a low baseline viral load, non-African-American race, and stage 0-2 fibrosisthat will predict a positive treatment response in patients. SVR12 rates should continue to be evaluated in patients receiving 8 weeks of LDV/SOF to ensure appropriate treatment.

\section{Conclusions}

The SVR12 rate obtained in this study is high, despite a large number of prescribers, variability in provider practice setting and specialty, differing fibrosis assessment methods, and timing of testing. The SVR12 rate was also similar to that obtained in other real-world analyses. This real-world, noncenter, community-based review of pharmacy records indicates that 8 weeks of LDV/SOF therapy in HCV GTl patients seems to be highly effective in real-world clinical practice. Reducing the duration of treatment could result in significant cost savings in appropriate patients and possibly allow more patients to receive
HCV treatment. Of note, the use of this approach in African Americans, especially with evidence of advanced fibrosis (stage 3 fibrosis), warrants discretion, as advised by the AASLD/ IDSA guidelines, and further clarification in future studies.

\section{Authors}

JENNIFER ANDRES, PharmD, BCPS, Department of Pharmacy Practice, Temple University School of Pharmacy, and KAMRAN QURESHI, MD, Section of Gastroenterology and Hepatology, Department of Medicine, Temple University Lewis Katz School of Medicine, Philadelphia, Pennsylvania. STEPHEN LOTT, PharmD, MS, CSP, Diplomat Specialty Pharmacy, Flint, Michigan.

AUTHOR CORRESPONDENCE: Jennifer Andres, PharmD, BCPS, Temple University School of Pharmacy, 3307 N. Broad St.,

Philadelphia PA 19140. Tel: 215.707.6014;

E-mail: jennifer.andres@temple.edu.

\section{DISCLOSURES}

No outside funding supported this study. Lott is an employee of Diplomat Pharmacy. Andres and Qureshi have nothing to disclose.

Study concept and design were contributed by Lott and Qureshi, who also collected the data. All authors contributed equally to data interpretation and manuscript preparation. Andres revised the manuscript, along with Lott and Qureshi.

This work was presented in part as a poster at the 2016 International Liver Conference; Barcelona, Spain; April 13-17, 2016.

\section{REFERENCES}

1. Gower E, Estes C, Blach S, Razavi-Shearer K, Razavi H. Global epidemiology and genotype distribution of the hepatitis $C$ virus infection. J Hepatol. 2014;61(1 Suppl):S45-57.

2. Mohd Hanafiah K, Groeger J, Flaxman AD, Wiersma ST. Global epidemiology of hepatitis $C$ virus infection: new estimates of age-specific antibody to HCV seroprevalence. Hepatology. 2013;57(4):1333-42.

3. Razavi H, Waked I, Sarrazin C, et al. The present and future disease burden of hepatitis $\mathrm{C}$ virus (HCV) infection with today's treatment paradigm. J Viral Hepat. 2014;21(Suppl 1):34-59.

4. Ly KN, Hughes EM, Jiles RB, Holmberg SD. Rising mortality associated with hepatitis C virus in the United States, 2003-2013. Clin Infect Dis. 2016;62(10):1287-88

5. Asselah T, Boyer N, Saadoun D, Martinot-Peignoux M, Marcellin P. Direct-acting antivirals for the treatment of hepatitis $C$ virus infection: optimizing current IFN-free treatment and future perspectives. Liver Int. 2016;36(Suppl 1):47-57.

6. van der Meer AJ, Veldt BJ, Feld JJ, et al. Association between sustained virological response and all-cause mortality among patients with chronic hepatitis C and advanced hepatic fibrosis. JAMA. 2012;308(24):2584-93.

7. American Association for the Study of Liver Disease, Infectious Diseases Society of America. HCV guidance: recommendations for testing, managing, and treating hepatitis C. Available at: http://www.hcvguidelines.org. Accessed November 19, 2017.

8. European Association for Study of Liver. EASL recommendations on treatment of hepatitis C 2016. J Hepatol. 2017;66(1):153-94. 
9. U.S. Food and Drug Administration, Center for Drug Evaluation and Research. Approval for sofosbuvir and ledipasvir fixed-dose combination. NDA 205834. 2014. Available at: https://www.accessdata.fda.gov/drugsatfda_ docs/nda/2014/205834Origls000Approv.pdf. Accessed December 6, 2017.

10. Harvoni (ledipasvir/sofosbuvir) tablets for oral use. Gilead Sciences. Revised November 2017. Available at: https://www.gilead.com/ /media/ Files/pdfs/medicines/liver-disease/harvoni/harvoni_pi.pdf. Accessed November 19, 2017.

11. Lawitz E, Poordad FF, Pang PS, et al. Sofosbuvir and ledipasvir fixed-dose combination with and without ribavirin in treatment-naive and previously treated patients with genotype 1 hepatitis $C$ virus infection (LONESTAR): an open-label, randomised, phase 2 trial. Lancet. 2014;383(9916):515-23.

12. Kowdley KV, Gordon SC, Reddy KR, et al. Ledipasvir and sofosbuvir for 8 or 12 weeks for chronic HCV without cirrhosis. N Engl J Med. 2014;370(20):1879-88

13. O’Brien TR, Feld JJ, Kottilil S, Pfeiffer RM. No scientific basis to restrict 8 weeks of treatment with ledipasvir/sofosbuvir to patients with hepatitis $C$ virus RNA<6,000,000 IU/mL. Hepatology. 2016;63(1):28-30.

14. Wilder JM, Jeffers LJ, Ravendhran N, et al. Safety and efficacy of ledipasvir-sofosbuvir in black patients with hepatitis $C$ virus infection: a retrospective analysis of phase 3 data. Hepatology. 2016;63(2):437-44.

15. O'Brien TR, Lang Kuhs KA, Pfeiffer RM. Subgroup differences in response to 8 weeks of ledipasvir/sofosbuvir for chronic hepatitis C. Open Forum Infect Dis. 2014;1(3):oful10.

16. Iyengar S, Tay-Teo K, Vogler S, et al. Prices, costs, and affordability of new medicines for hepatitis $\mathrm{C}$ in 30 countries: an economic analysis. PLoS Med. 2016;13(5):e1002032.

17. Saab S, Jimenez M, Fong T, et al. Accessibility to oral antiviral therapy for patients with chronic hepatitis C in the United States. J Clin Transl Hepatol. 2016;4(2):76-82.

18. Chhatwal J, He T, Hur C, Lopez-Olivo MA. Direct-acting antiviral agents for patients with hepatitis $C$ virus genotype 1 infection are cost saving. Clin Gastroenterol Hepatol. 2017;5(6):827-37.

19. Younossi ZM, Park H, Saab S, Ahmed A, Dieterich D, Gordon SC. Cost-effectiveness of all-oral ledipasvir/sofosbuvir regimens in patients with chronic hepatitis $\mathrm{C}$ virus genotype 1 infection. Aliment Pharmacol Ther. 2015;4l(6):544-63.
20. Terrault NA, Zeuzem S, Di Bisceglie AM, et al. Treatment outcomes with 8,12 and 24 week regimens of ledipasvir/sofosbuvir for the treatment of hepatitis $\mathrm{C}$ infection: analysis of a multicenter prospective, observational study. Hepatology. 2015;62(1 Suppl):256A [Abstract]. Available at: http:// www.natap.org/2015/AASLD/AASLD_04.htm. Accessed November 19, 2017.

21. Lai JB, Witt MA, Witt DJ. Real-world effectiveness of 8, 12 and 24 weeks of ledipasvir (ldv)/sofosbuvir(sof)-based therapy for hepatitis C (HCV) genotype 1: analysis in a large integrated health care system. J Hepatol. 2016;64 (2 Suppl):S778 [Abstract SAT-177]. Available at: http://www.journal-ofhepatology.eu/article/S0168-8278(16)01517-8/abstract. Accessed November 19, 2017.

22. Buggisch B, Peterson J, Mauss S, et al. Ledipasvire/sofosbuvir (LDV/SOF) for 8 wks in genotype 1 (GTl) treastment-naive non-cirrhotic patients with HCV viral load < 6 million IU/ML $(6 \mathrm{M})$ : a comparative analysis of the phase3 ION-3 data and real world effectiveness. J Hepatol. 2016;64(2 Suppl):S810 [Abstract SAT-242]. Available at: http://www.journal-of-hepatology.eu/ article/S0168-8278(16)01581-6/fulltext. Accessed November 19, 2017.

23. Latt NL, Gevorkyan R, Yanny BT, Sahota A. Ledipasvir/sofosbuvir for 8 weeks in non cirrhotic treatment naive patients with genotype 1 hepatitis $C$ infection: real life experience in a community setting. J Hepatol. 2016;64 (2 Suppl):S802 [Abstract SAT-227]. Available at: http://www.journal-ofhepatology.eu/article/S0168-8278(16)01567-1/abstract. Accessed November 19, 2017.

24. Marshall VJ, Rife K, Hirsch A, et al. Real life treatmet outcomes with 8 week course treatment for hepatitis $C$ without cirrhosis confirmed by transient elastography. Hepatology. 2015;62(1 Suppl):779A [Abstract 1154]. Available at: http://liverlearning.aasld.org/aasld/2015/thelivermeeting/110398/anita.moreland.real.life.treatment.outcomes.with.8.week.course. treatment.for.html. Accessed November 19, 2017.

25. Roytman M, Tang AL, Wu C, et al. Real life experience with sofosbuvir and ledipasvir fixed dose regimen in the multiethnic cohort of patients with chronic hepatitis C. Hepatology. 2015;62(1 Suppl):761A [Abstract 1121]. Available at: https://liverlearning.aasld.org/aasld/2015/thelivermeeting/110365/alister.tang.real.life.experience.with.sofosbuvir.and.ledipasvir. fixed.dose.html. Accessed November 19, 2017.

26. Crespo J, Fenandez I, Cabezas J, et al. Effectiveness and safety of sofosbuvir/ledipasvir treatment for monoinfected genotype $1 \mathrm{HCV}$ patients in real life clinical practice: results of the Spanish Hepa-C cohort. J Hepatol. 2016;64(2 Suppl):S217 [Abstract LBP511]. Available at: http://www.journal-of-hepatology.eu/article/S0168-8278(16)00188-4/abstract. Accessed November 19, 2017.

27. Kowdley KV, Sundaram V, Jeon CY, et al. Eight weeks of ledipasvir/ sofosbuvir is effective for selected patients with genotype 1 hepatitis $C$ virus infection. Hepatology. 2017;65(4):1094-103. 